\title{
Antimalarial Activity of Various Bisbenzylisoquinoline and Aporphine- Benzylisoquinoline Alkaloids and their Structure-Activity Relationships against Chloroquine - Sensitive and Resistant Plasmodium falciparum Malaria in vitro
}

Zuguang Ye $\mathrm{e}^{1,2}$ and Knox Van Dyke ${ }^{2^{*}}$

${ }^{1}$ Institute of Chinese Materia Medica, China Academy of Chinese Medical Sciences; 16 Nanxiaojie St. Dongzhimen, Beijing, China

2Department of Biochemistry and Molecular Pharmacology West Virginia University Medical Center, Med Center Dr. Morgantown W.V, 26506, USA

*Corresponding author: Knox Van Dyke, Professor, West Virginia University Health Sciences Center, Biochemistry/Molecular Pharmacology, 1 Med Center Drive, Morgantown, WV 26506, USA, Tel: 0013042935449; Fax: 001304 2936846; E-mail: kvandyke@hsc.wvu.edu

Rec date: Nov 26, 2015; Acc date: Dec 5, 2015; Pub date: Dec 12, 2015

Copyright: ( 2015 Ye Z, et al. This is an open-access article distributed under the terms of the Creative Commons Attribution License, which permits unrestricted use, distribution, and reproduction in any medium, provided the original author and source are credited.

\begin{abstract}
The antimalarial activity of some bisbenzylisoquinoline (BBIQ) alkaloids were studied using in vitro culture of both chloroquine (CQ)-sensitive and chloroquine-resistant strains of Plasmodium falciparum. The combination of chloroquine and tetrandrine gave a 44 fold potentiation of malarial killing. Fangchinoline, hernandezine, pycnamine, berbamine and isotetrandrine had similar antimalarial activity as tetrandrine against the sensitive strain of $P$. falciparum malaria. Hernandezine, isotetrandrine, berbamine, fangchinoline and methoxyadiantifoline had similar antimalarial activity as tetrandrine against a strain of chloroquine-resistant falciparum malaria. Based on structure/ activity analysis, we concluded that the stereochemistry of C-1' (S ring) of the BBIQ structure played an important role in selective action against resistant malaria. The stereochemistry of C-1 (left ring) and a substituent of C-12 appeared to have little influence on the selectivity. The point of attachment of the ether bridges to the rings also exerts an important influence on selective antimalarial activity. Tetrandrine (TT) penetrates both the erythrocyte and parasite membranes and inhibits the synthesis and activity of the (MDR) pump and likely the Plasmodium falciparum chloroquine resistance transporter (Pfcrt) since it inhibits the calcium channel. These observations likely account for the fact TT potentiates the activity of chloroquine more than 40 fold against chloroquine resistant falciparum malaria.
\end{abstract}

Keywords: Chloroquine sensitive and resistant falciparum malaria; Bisbenzylisoquinolines; Structure-activity; Tetrandrine; MDR: multiple drug resistance; Pfcrt: Plasmodium falciparum resistance transporter

\section{Abbreviations: \\ MDR: Multiple Drug Resistance; PfCRT: Plasmodium falciparum Resistance Transporter; ME: Methoxyadiantifoline}

\section{Background}

Our previous work revealed that tetrandrine (TT) possessed surprising antimalarial activity, because it exerted a selective action against the chloroquine-resistant strain of P. falciparium malaria in vitro [1]; TT was also found to greatly potentiate antimalarial activity of chloroquine (CQ) or qinghaosu (artemesinin) when used in combination [2]. The purpose of this paper is to delineate several important structure-activity relationships through the investigation of the antimalarial activity of several compounds structurally similar to tetrandrine (Figure 1). Absolute antimalarial activity relative to TT was also measured. Since Cepharanthine (CE) had direct anticancer activity [3] and caused reversal of MDR resistance in cancer [4,5], it was tested for MDR reversal activity and for possible potentiating effect on antimalarial activity. In addition, two aporphinebenzylisoquinoline alkaloids - thalicarpine (TH) and methoxyadiantifoline (ME) - were selected for testing. The former reportedly has antitumor activity [6], increased the antitumor effect of cyclophosphamide when used in combination [7], and affected the cardiovascular system [8]. ME is a relatively new alkaloid isolated from Thalictrum omeiense [9] which affects the cardiovascular system by increasing coronary blood flow and producing negative inotropic, chronotropic [10], and antiarrhythmic effects [11]. These biological activities likely result from inhibition of calcium channels $[10,11]$. Owing to similarities in chemical structure and biological activities between these alkaloids and bisbenzylisoquinolines; e.g., TT, CE and hernandezine (HE), were investigated for antimalarial activities of TH and ME against a chloroquine sensitive and a resistant strain of $P$. falciparum malaria.

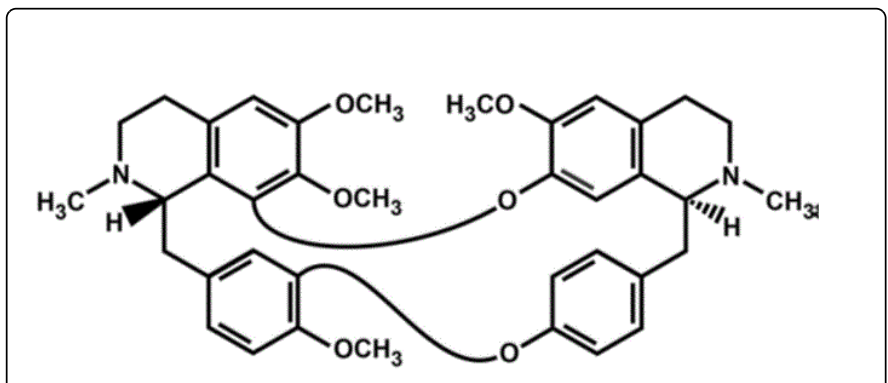

Figure 1: The chemical structure of tetrandrine. 
Citation: Ye Z, Van Dyke K (2015) Antimalarial Activity of Various Bisbenzylisoquinoline and Aporphine-Benzylisoquinoline Alkaloids and their Structure-Activity Relationships against Chloroquine - Sensitive and Resistant Plasmodium falciparum Malaria in vitro. Malaria Contr Elimination 5: 137. doi:10.4172/2470-6965.1000137

Page 2 of 7

\section{Materials and Methods}

ME was kindly supplied by Dr. Chang-geng Mo. Cepharanthine was supplied by the National Cancer Institute. Because the bisbenzylisoquinoline alkaloids are insoluble in water at $\mathrm{pH}=7$, we added several drops of $1.0 \mathrm{~N}$ hydrochloride acid to dissolve the alkaloids. The $\mathrm{pH}$ of this solution was adjusted to about 6.0 by adding sufficient $1.0 \mathrm{~N} \mathrm{NaOH}$. Other alkaloid stock solutions $\left(1 \times 10^{-2} \mathrm{M}\right)$ were produced similarly. After sterilization by filtration, serial dilutions of the alkaloids were made using the complete medium with $10 \%$ pooled human A+ serum, $25 \mathrm{mM}$ HEPES and $25 \mathrm{mM} \mathrm{NaHCO}_{3}$, prior to each experiment.

Two strains of $\mathrm{P}$ falciparum were used in these experiments: the CQ-sensitive FCMSU1/Sudan strain was donated [12] and the CQresistant Indochina (W2) strain was provided by Dr. W.K. Milhous. Both parasite strains were cultured in our lab for over two years using the candle jar method [12]. For a given experiment, 4-day-old Petri dish cultures with 5-10\% parasitemia were diluted with medium containing sufficient non-parasitized human type A erythrocytes to obtain a culture with a final hematocrit of $1.5 \%$ and parasitemia of $0.5-1.0 \%$.

Antimalarial activity of various compounds was assessed using the method of Desjardins et al. [13]. Each well of flat-bottomed, plastic, 96-well microtiter plates contained a total volume of $250 \mu \mathrm{l}$ of complete medium with or without the alkaloid to be tested; [2] $200 \mu \mathrm{l}$ of either the parasitized culture or non-parasitized human erythrocytes as a control; and (3) after incubation of the plates in a candle jar for $24 \mathrm{hr}$ at $37^{\circ} \mathrm{C}, 25 \mu \mathrm{l}$ of $(2,8-3 \mathrm{H})$ adenosine $(0.1 \mu \mathrm{Ci})$ were added to each well. Plates were incubated at $37^{\circ} \mathrm{C}$ for an additional $18 \mathrm{hr}$. Afterwards, the contents of the well were harvested onto small filter disks of fiberglass using a Bellco semi-automated cell harvester (BSCH). The filters were washed in the BSCH five times with distilled water and each disk was removed and placed into a standard $20 \mathrm{ml}$ glass liquid scintillation vial containing $10 \mathrm{ml}$ of aqueous based liquid scintillation fluid [14]. Radioactivity was counted using a Packard Tri-Carb scintillation spectrometer Model 2425. The inhibitory concentration at 50\% response [15] IC50 of a compound was calculated using probits. Duplicate wells were prepared for each concentration of drugs. Experiments were repeated three times.

\section{Results and Discussion}

In Table 1 , the surprising synergistic effect ( 44 fold) can be seen with the combination of tetrandrine and chloroquine versus chloroquine alone against the chloroquine resistant strain of falciparum malaria by comparing their respective inhibitory concentration at $50 \%$ response (IC 50). The data* in the Table 1 represents the mean value $+/$ - the standard deviation (nM) from three experiments. Ratios of TT/CQ ${ }^{* *}$ in the drug combinations are in 10:3, $10: 1$, and $30: 1$, respectively. ${ }^{* *} \mathrm{~S}$ and $\mathrm{R}$ strains represent CQ-sensitive strains (FCMSU1/ (Sudan) and CQ resistant (W2) strain of $P$. falciparum malaria, respectively. The purpose of displaying this data is that it represents the synergistic power of the correct combinations of chloroquine and terandrine against a chloroquine resistant strain of falciparum malaria. Table1 represents the comparative control to measure the other various bisbenzylisoquinoline (BBIQ) structures against the tetrandrine and chloroquine combination. Most of the other drugs have similar potentiating activity but tetrandrine is probably the drug of choice to accompany chloroquine to reverse chloroquine resistance since it is very effective against chloroquine resistance with little toxicity at the low concentrations utilized. In Table 2 the data are expressed as the ratio of the iC50's where St/Rt where St is the chloroquine sensitive malarial strain and $\mathrm{Rt}$ is the chloroquine resistant malarial strain. If the IC50 of the resistant strain is large relative to the sensitive strain the final ratio would be a fraction. If the sensitive strain is large, and resistant strain is small it would produce a larger whole number as the ratio. The best idea is to decrease the resistance to a small IC50 and have chloroquine sensitivity a small number as well. This would mean both strains of malaria parasites could be treated with lower doses of these combinational drugs. In general, low doses produce less toxicity which is generally helpful to the patient.

\begin{tabular}{|l|l|l|l|l|l|}
\hline \multicolumn{2}{|l|}{ Single Drug } & \multicolumn{2}{l|}{ Drug Combination } \\
\hline Malaria*** & TT & CQ & $\begin{array}{l}\text { TT }(1.0 \mu \mathrm{M}) \\
\mathrm{CQ}(0.3 \mu \mathrm{M})\end{array}$ & $\begin{array}{l}\text { TT }(2.0 \mu \mathrm{M}) \\
\text { CQ }(0.2 \mu \mathrm{M})\end{array}$ & $\begin{array}{l}\text { TT }(3.0 \mu \mathrm{M}) \\
\mathrm{CQ}(0.1 \mu \mathrm{M})\end{array}$ \\
\hline S Strain & $498.1 \pm 93.7$ & $26.7 \pm 3.8$ & $\begin{array}{l}54.9 \pm 7.1(\mathrm{TT}) \\
16.5 \pm 2.1(\mathrm{CQ})\end{array}$ & $\begin{array}{l}114.1 \pm 23.0(\mathrm{TT}) \\
11.4 \pm 2.3(\mathrm{CQ})\end{array}$ & $\begin{array}{l}223.3 \pm 38.6(\mathrm{TT}) \\
7.4 \pm 1.3(\mathrm{CQ})\end{array}$ \\
\hline R Strain & $197.5 \pm 24.7$ & $185.8 \pm 4.9$ & $\begin{array}{l}79.5 \pm 13.7(\mathrm{TT}) \\
23.8 \pm 4.1(\mathrm{CQ})\end{array}$ & $\begin{array}{l}79.5 \pm 16.1(\mathrm{TT}) \\
8.0 \pm 1.6(\mathrm{CQ})\end{array}$ & $\begin{array}{l}124.6 \pm 9.6(\mathrm{TT}) \\
4.2 \pm 0.3(\mathrm{CQ})\end{array}$ \\
\hline
\end{tabular}

Table 1: IC50 (nM) of TT (Tetrandrine) and CQ (Chloroquine) for each drug alone and in combination ${ }^{*}{ }^{*}$ The data in the table above are mean values \pm S.D. $(\mathrm{nM})$ from three experiments. ${ }^{* *}$ Ratios of TT/CQ in the drug combination are 10:3, 10:1, 30:1, respectively. ${ }^{* *}$ S and R strains represent CQ-sensitive (FCMSU1.Sudan) and resistant (w2) strains of $P$. falciparum, respectively.

The general structure of a bisbenzylisoquinoline alkaloid is shown in Figure 2. Note that the chiral carbons are labeled " $\mathrm{C} 1$ " " on the right side carbon and "Cl" on the left side. 


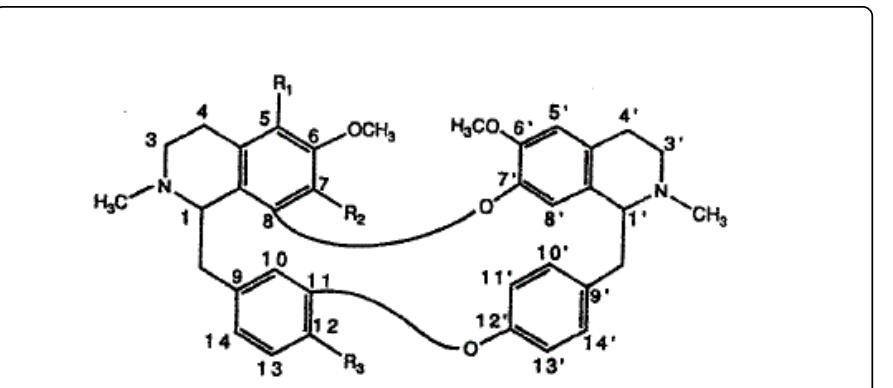

Figure 2: The general structure of bisbenzylisoquinoline alkaloids with numbering.

The determination of R and S configuration at C-1 and C-1' follow rules proposed by Cahn et al., [16]. Priority of four different atoms or groups attached to a chiral center follow the atomic number and the atom with the highest atomic number has the highest priority. We see that the chiral center with the lower priority group is directed away from us and observe the arrangement of the remaining groups. Proceeding from groups in descending priority, our eye travels in a clockwise direction, and the configuration is $\mathrm{R}$. If the eye travels in a counter clockwise direction, the configuration is S (Figure 3).

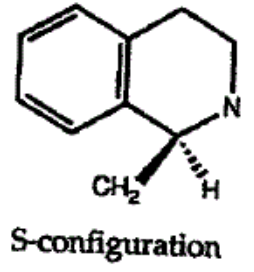

Figure 3: R,S configurations applied to $\mathrm{C}-1$ ' benzylisoquinoline (right side ring).

The $\mathrm{S}$ form at $\mathrm{C}-1^{\prime}$ has the hydrogen projecting behind the paper and the $\mathrm{CH} 2$ (carbon attached between $\mathrm{C}-1^{\prime}$ and the $\mathrm{C}-9^{\prime}$ ) projects forward. In the $\mathrm{R}$ form, the hydrogen projects forward and the $\mathrm{CH} 2$ projects beneath the plane of the paper.

As seen in Table 1, TT, isotetrandrine (IT), HE and berbamine (BB) had potent and selective antimalarial activity against CQ-resistant $P$. falciparum malaria in vitro. Selectivity is measured by the ratio produced by dividing the IC50 value for the sensitive strain by the IC50 value of the resistant strain. The ratios of four aforementioned alkaloids ranged from 2.6 to 3.5 ; all were $\mathrm{S}$ configuration at $\mathrm{C}-1^{\prime}$. When the alkaloid stereochemistry of $\mathrm{C}-1^{\prime}$ is $\mathrm{R}$, the selective antimalarial action against CQ resistance was not displayed. The ratios of pycnamine (PY) and phaeanthine (PH) with the $\mathrm{R}$ configuration at $\mathrm{C}-1^{\prime}$ was 0.9 and 1.2 respectively, although their chemical structures were identical to those of $\mathrm{BB}$ and IT except for the stereochemistry of C-1'. Based on the comparison of IC50 values between BB and PY, or IT and PH, we see that the IC50 values of PY and PH against the CQresistant malaria were 2-3 fold higher than those of $\mathrm{BB}$ and IT. However, the IC50 values of PY and PH for the CQ-sensitive malaria were very close to those of $\mathrm{BB}$ and IT. As a result, there was not a significant difference in antimalarial activity between the sensitive and resistant malaria strains. Therefore, the sensitivity of the CQ-resistant strain of $P$. falciparum to $\mathrm{PY}$ and $\mathrm{PH}$ was decreased and the selectivity of PY and PH was lost with the change of the stereochemistry at C-1'.

Although the stereochemistry of C-1' played an important role in the selective antimalarial activity of a bisbenzylisoquinoline alkaloid, the configuration at $\mathrm{C}-1$ (the left side rings) appeared to have little influence on the selectivity. As shown in Table 1, both IT and BB have a $\mathrm{R}$ configuration at $\mathrm{C}-1$ position, but they still maintained the antimalarial selectivity as did TT with the $\mathrm{S}$ configuration at C-1' because of their larger ratios (3.5 for IT and 2.6 for $\mathrm{BB}$ ) than TT [26]. Thus we suggest that the configuration at C-1 contributes little to the antimalarial selectivity. Apparently, the stereochemistry of C-1' but not C-1 was critical for the selective antimalarial activity of the bisbenzylisoquinoline alkaloids tested.

A substituted methoxyl group at "R2" (Figure 2) demonstrated remarkable influence on the antimalarial selectivity. We showed that substances exhibiting selective activity against CQ-resistant $P$ falciparum malaria had a methoxyl group at the R2 position, while the alkaloids like obamegine (OB) and fangchinoline (FA) having a hydroxyl group at R2 displayed weak selectivity toward chloroquineresistance. If we compared the ratios of $\mathrm{OB}$ and $\mathrm{BB}$ or FA and TT (each above pair of compounds shared the same chemical structure except for a substituent, methoxyl or hydroxyl group at R2), it was apparent that the ratios of OB and FA (1.4 for OB and 1.2 for FA) were lower than those of BB and TT. Selective antimalarial activity was greatly affected by whether the substituent at R2 was a methoxyl group or a hydroxyl group. However, a substituent, methoxyl group and hydroxyl group, at R3 (Figure 2) exerts little change in the antimalarial selectivity of the alkaloids because the ratio of $\mathrm{BB}(2.6)$ was close to the ratio of IT (3.5). Chemical structures of BB and IT are identical except for different substituents of R3; i.e., BB had a hydroxyl group at R3, wheras IT had a methoxyl group at R3. Nevertheless, both exhibit identically potent antimalarial selectivity. Therefore, the selective antimalarial activity of bisbenzylisoquinoline alkaloids might well depend on the substitution of a methoxyl group at R2, but not at R3.

Kuroda et al. studied the antitumor effect of 23 different bisbenzylisoquinoline alkaloids [6]. With the exception of CE, they concluded that $\mathrm{S}$ configuration at $\mathrm{C}-1$ and $\mathrm{C}-1^{\prime}$ was a chemical structure requirement for antitumor activity. The $\mathrm{S}$ configuration had influence on antitumor activity whether substituents at R2 and R3 or C-12' were hydroxyl or methoxyl group. Russian scientists tested 15 bisbenzylisoquinoline alkaloids for tuberculostatic activity [17] and discovered that the tuberculostatic activity relied on the configuration at the asymmetric centers. Our results differed somewhat. Based on the data in this study, $\mathrm{S}$ configuration at $\mathrm{C}-1^{\prime}$ alone played a crucial role in manifestation of the antimalarial selectivity against chloroquineresistance. This may reflect a biological difference between malaria parasites and cancer cells [17] even though the MDR protein is similar in genetic composition [18] and molecular weight [19]. All groups realized that a methoxyl group at C-7 was an important structural requirement of bisbenzylisoquinolines to exhibit biological activity $[6,17]$.

FA with an IC50 of $2.6 \times 10-7 \mathrm{M}$ and TT with an IC50 of $2.9 \mathrm{x}$ $10-7 \mathrm{M}$ possessed the most potent antimalarial activity against CQsensitive $P$. falciparum malaria, while TT, HE and IT had the most potent activity against the CQ-resistant malaria with IC50 of $1.2 \mathrm{x}$ 10-7, $1.3 \times 10-7$ and $1.4 \times 10-7$, respectively. Partridge et al. investigated $\mathrm{PH}, \mathrm{PY}$ and other alkaloids for antimalarial activity using a MDR strain of $P$. falciparum in vitro [20]. They demonstrated that IC50 
Citation: Ye Z, Van Dyke K (2015) Antimalarial Activity of Various Bisbenzylisoquinoline and Aporphine-Benzylisoquinoline Alkaloids and their Structure-Activity Relationships against Chloroquine - Sensitive and Resistant Plasmodium falciparum Malaria in vitro. Malaria Contr Elimination 5: 137. doi:10.4172/2470-6965.1000137

Page 4 of 7

values for PH and PY were $1.43 \mu \mathrm{g} / \mathrm{ml}$ about $2.3 \times 10^{-6} \mathrm{M}$ ) and 0.15 $\mu \mathrm{g} / \mathrm{ml}$ (about $2.5 \times 10^{-7} \mathrm{M}$ ), respectively. The IC50 of PY that we found was higher than theirs, while the IC50 of $\mathrm{PH}$ was much lower than theirs. A difference in strain or experimental conditions might partially account for the difference in antimalarial efficacy between the two laboratories (Table 2).

\begin{tabular}{|c|c|c|c|c|c|c|c|c|c|c|}
\hline \multirow{2}{*}{$\begin{array}{l}\text { Drug } \\
\text { (a) } \\
\mathrm{TT}\end{array}$} & \multicolumn{2}{|c|}{$\begin{array}{l}\text { Configuration } \\
\text { C-1 C-1' }\end{array}$} & \multicolumn{3}{|c|}{$\begin{array}{l}\text { Substituents } \\
\text { R1 R2 R3 }\end{array}$} & \multirow{2}{*}{$\begin{array}{l}\text { See Figuret } \\
\text { 1or2 }\end{array}$} & \multirow{2}{*}{$\begin{array}{l}\begin{array}{l}\text { Oxygen } \\
\text { Bridge(s) }\end{array} \\
\text { C8-C7' } \\
\text { C11-12' }\end{array}$} & \multicolumn{2}{|c|}{$\begin{array}{l}\text { IC50(10-7M) } \\
\mathrm{St}^{\star \star} \mathrm{Rt}^{\star \star}\end{array}$} & \multirow{2}{*}{$\begin{array}{l}\text { Ratio St/Rt• } \\
2.5\end{array}$} \\
\hline & $S$ & $S$ & $\mathrm{H}$ & $\mathrm{OCH} 3$ & О3CH3 & & & 2.9 & 1.2 & \\
\hline IT & $\mathrm{R}$ & $S$ & $\mathrm{H}$ & $\mathrm{OCH} 3$ & $\mathrm{OCH} 3$ & 2 & $\begin{array}{l}\mathrm{C} 8-\mathrm{C} 7^{\prime} \\
\mathrm{C} 11-\mathrm{C} 12^{\prime}\end{array}$ & 4.8 & 1.4 & 3.5 \\
\hline HE & $S$ & $S$ & $\mathrm{OCH} 3$ & $\mathrm{OCH} 3$ & $\mathrm{OCH} 3$ & 2 & $\begin{array}{l}\mathrm{C} 8-\mathrm{C} 7^{\prime} \\
\mathrm{C} 11-\mathrm{C} 12^{\prime}\end{array}$ & 3.7 & 1.3 & 2.8 \\
\hline BB & $\mathrm{R}$ & $S$ & $\mathrm{H}$ & $\mathrm{OCH} 3$ & $\mathrm{OH}$ & 2 & $\begin{array}{l}\mathrm{C} 8-\mathrm{C} 77^{\prime} \\
\mathrm{C} 11-\mathrm{C} 12^{\prime}\end{array}$ & 4.6 & 1.9 & 2.6 \\
\hline PY & $\mathrm{R}$ & $\mathrm{R}$ & $\mathrm{H}$ & $\mathrm{OCH} 3$ & $\mathrm{OH}$ & 2 & $\begin{array}{l}\mathrm{C} 8-\mathrm{C} 7^{\prime} \\
\mathrm{C} 11-\mathrm{C} 12^{\prime}\end{array}$ & 3.8 & 4.2 & 0.9 \\
\hline $\mathrm{PH}$ & $\mathrm{R}$ & $\mathrm{R}$ & $\mathrm{H}$ & $\mathrm{OCH} 3 \mathrm{H}$ & $\mathrm{OCH} 3$ & 2 & $\begin{array}{l}\mathrm{C} 8-\mathrm{C} 7{ }^{\prime} \\
\mathrm{C} 11-\mathrm{C} 12^{\prime}\end{array}$ & 6.0 & 5.0 & 1.2 \\
\hline OB & $\mathrm{R}$ & $S$ & $\mathrm{H}$ & $\mathrm{OH}$ & $\mathrm{OH}$ & 2 & $\begin{array}{l}\mathrm{C} 8-\mathrm{C} 7^{\prime} \\
\mathrm{C} 11-\mathrm{C} 12^{\prime}\end{array}$ & 6.6 & 4.8 & 1.4 \\
\hline FA & $S$ & $S$ & $\mathrm{H}$ & $\mathrm{OH}$ & $\mathrm{OCH} 3 \mathrm{H}$ & 2 & $\begin{array}{l}\mathrm{C} 8-\mathrm{C} 7^{\prime} \\
\mathrm{C} 11-\mathrm{C} 12^{\prime}\end{array}$ & 2.6 & 2.2 & 1.2 \\
\hline ME & $S$ & $S$ & $\mathrm{OCH} 3$ & -- & -- & 7 & C10-C9' & 53 & 9.7 & 5.5 \\
\hline $\mathrm{TH}$ & $S$ & $S$ & $\mathrm{H}$ & -- & -- & 7 & C10-C9' & 17 & 13 & 1.3 \\
\hline $\mathrm{CY}$ & $\mathrm{R}$ & $\mathrm{R}$ & -- & -- & -- & 4 & $\begin{array}{l}\mathrm{C} 8-\mathrm{C} 12^{\prime} \\
\mathrm{C} 12-\mathrm{C} 8^{\prime}\end{array}$ & 32 & 42 & 0.8 \\
\hline CE & $\mathrm{R}$ & $S$ & -- & -- & -- & 5 & $\begin{array}{l}\mathrm{C} 8-\mathrm{C} 7^{\prime} \\
\mathrm{C} 12-\mathrm{C} 11^{\prime}\end{array}$ & 10 & 9.4 & 1.1 \\
\hline
\end{tabular}

Table 2: Chemical, Structure-Antimalarial Activity of Bisbenzylisoquinoline Alkaloids against Plasmodium falciparum in vitro. TT-tetrandrine; IT-isotetrandrine; HE-hernandezine; BB-berbamine; PY-pycnamine; PH-phaeanthine; OB-obamegine; FA-fangchinoline; CY-cycleanine; CEcepharanthine; ME-methoxyadiantifoline; TH-thalicarpine. The IC50 of a drug against sensitive strain of $P$. falciparum is divided by the IC50 for the same drugs against the resistant strain. ${ }^{*}$ St and Rt represent chloroquine-sensitive and resistant strain of $P$. falciparum. $\dagger$ See the indicated figure to determine the structure.

The connections of the ether bridges in the bisbenzylisoquinoline structures play a role in antimalarial activity. As shown in Figure 2, the first eight compounds had two ether bridges connecting the ring structures on the right from ring structures on the left. The linkage of the two ether bridges were from C-8 to C-7' and C-11 to C-12' and are termed "head-head" and "tail-tail" connections, respectively. The connections of the ether bridges of $\mathrm{CY}$ were $\mathrm{C}-8$ to $\mathrm{C}-12^{\prime}$ and $\mathrm{C}-12$ to C-8' (Figure 4); these are termed "head-tail" connections. The connection for CE was from C-8 to C-7' and C-12 to C-11' are termed "head-head" (Figure 5). The IC50 values for CY and CE are higher than the first eight compounds. Previously Partridge et al. [20] noted the attachment of ether bridges plays a role in antimalarial activity of bisbenzylisoquinoline alkaloids. These observations are summarized in Figure 6.

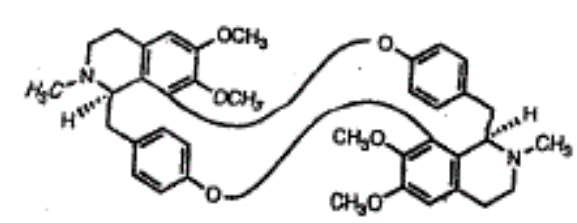

Figure 4: The structure of cycleanine. 
Citation: Ye Z, Van Dyke K (2015) Antimalarial Activity of Various Bisbenzylisoquinoline and Aporphine-Benzylisoquinoline Alkaloids and their Structure-Activity Relationships against Chloroquine - Sensitive and Resistant Plasmodium falciparum Malaria in vitro. Malaria Contr Elimination 5: 137. doi:10.4172/2470-6965.1000137

Page 5 of 7

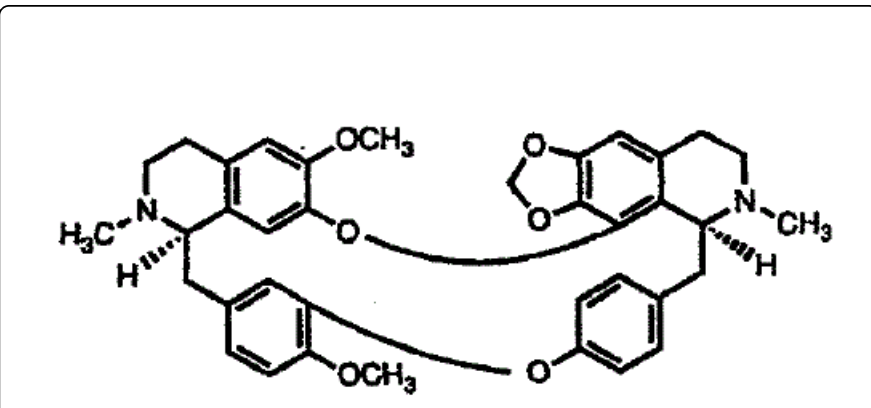

Figure 5: The structure of cepharanthine.

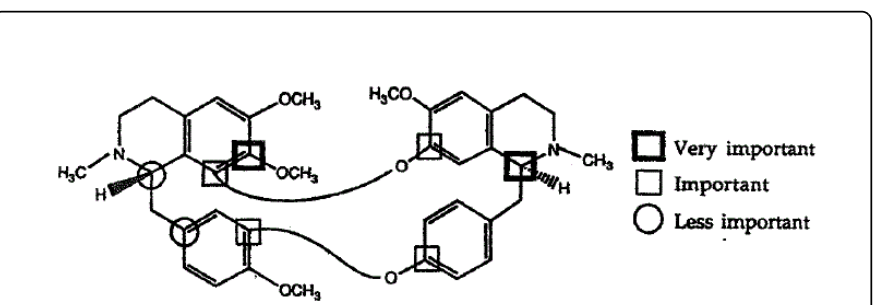

Figure 6: Summary of the important areas of the tetrandrine structure for selective antimalarial activity against chloroquine resistant falciparum malaria.

Tolkachev et al. found that TT and oxyacanthine were the most effective in tuberculostatic activity, while CY was inactive [17]. In this study, TT was considered to be one of the most potent anti-malarial alkaloids and CY one of the least. There were similarities between the experimental results of ours and theirs, although the two experimental systems are totally different. In addition, some work showed that TT was highly effective against tuberculosis in mice, even against the resistant tuberculosis strain [21]. Those results revealed that there were some common features between malaria parasites and tubercle bacillus in their sensitivities to TT or other bisbenzylisoquinoline alkaloids [22].

Like many calcium channel inhibitors, some bisbenzylisoquinoline alkaloids including aporphine-benzylisoquinoline alkaloids - had biological action on the cardiovascular system (which were roughly correlated with calcium channel antagonism). In addition, verapamil was found to have a selective antimalarial activity against drug resistant malaria [23]. Apparently, there was a correlation between their antimalarial selectivity and their calcium antagonism. However, the dependency of their selective activity on calcium channels blockade was partly serendipitous since our previous work demonstrated that the selective antimalarial activity to stereoisomers of verapamil and its derivatives was independent of calcium channels [23]. It is a fact that all calcium channel inhibiting drugs do not reverse multiple drug resistance. However, if a drug is a hydrophobic calcium channel inhibitor with the correct stereochemistry there is a clear possibility that chloroquine resistance in malaria can be overcome and/or reversed.

Besides bisbenzylisoquinoline alkaloids, two aporphinebenzylisoquinoline alkaloids, ME and TH, were also studied (Figure 7). While both exhibited antimalarial activity, the IC50's of ME and TH were much higher than the bisbenzylisoquinoline alkaloids. Therefore, the chemical structure of most bisbenzylisoquinoline alkaloids seems to favor more potent antimalarial activity as compared to aporphinebenzylisoquinolines. Although ME possessed the greatest ratio of all tested alkaloids, it was actually the least effective of all the substances tested against the CQ-sensitive P. falciparum with an IC50 for the CQsensitive malaria of $5.3 \times 10^{-6} \mathrm{M}$. This concentration is considerably higher than other compounds tested and thus ME was judged to be a comparatively weak antimalarial, although it displayed selective action toward CQ resistance.

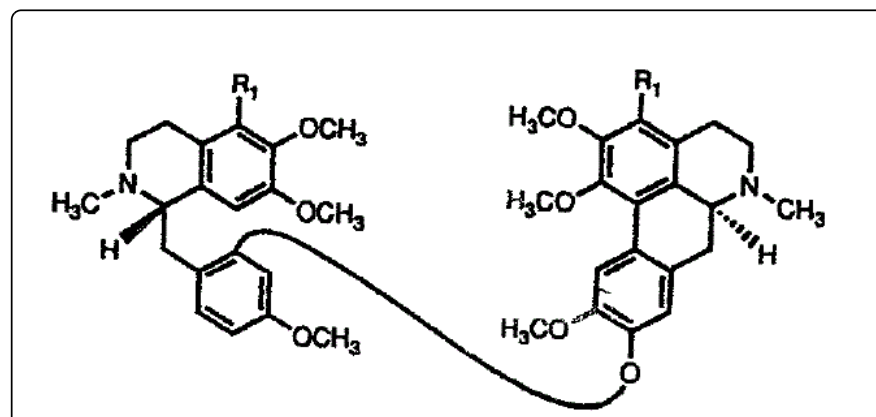

Figure 7: The general structure of benzylisoquinoline aporphine alkaloids.

Considering the IC50 values of alkaloids studied, the most effective antimalarial was TT. Some alkaloids e.g., IT, HE and FA, exhibit similar antimalarial efficacy. Therefore, IT, HE and FA, as well as BB and CE are considered to be worthy of study for further antimalarial activity.

A major difference between chloroquine-sensitive and resistant malaria parasites is the concentration of the MDR protein. This protein functions as an exit pump for chloroquine. The resistant cell overexpresses this protein 3-4 fold compared to sensitive strains and this prevents chloroquine lethality in this parasite. Naturally this is a selective advantage for the resistant parasite in a chloroquine environment. If a drug exerts a selective action toward the chloroquine-resistant parasite, the possibility exists that the drug uses the MDR protein to enter some portion of the cell [1]. Furthermore, when a bisbenzylisoquinoline - such as tetrandrine at micromolar antimalarial concentration - is combined with chloroquine, a striking synergism with chloroquine action occurs. In fact, $3 \mu \mathrm{M}$ tetrandrine decreases the IC50 of chloroquine 43 fold. The chloroquine-resistant parasite becomes more sensitive to chloroquine than the chloroquine sensitive parasite! Why should this occur? Probably when the exit of chloroquine in the resistant parasite is partially prevented, the real toxicity to chloroquine is displayed.

We think the comparison between the MDR system in cancer and malaria cells is striking. The genetic sequence and molecular weight of the MDR protein is similar in both diseases, and the drug binding to the MDR channel also appears to be similar. Therefore, it should not be surprising to see a parallelism between MDR sensitivity in malaria and cancer. In addition, MDR sensitivity in drug resistant TB appears be similar to malaria and cancer as well.

Recently, we have demonstrated that tetrandrine (a bisbenzyl isoquinoline) in combination with chloroquine can cure chloroquineresistant-falciparum malaria in Aotus monkeys if given over a several week period [24]. Since we know that tetrandrine can boost the antimalarial activity of chloroquine over 40 fold in a chloroquineresistant falciparum malarial strain [2], and since tetrandrine has a 
long biological half-life (weeks) in man and monkeys, our studies in Aotus should not be particularly surprising. In addition, we demonstrated that a fourfold increase $(4 \mathrm{x})$ multiple drug resistant (MDR) pump exists in the falciparum malaria parasite with chloroquine-resistance. The MDR glycoprotein displays a molecular weight similar to that in MDR cancer. We know that both chloroquine and tetrandrine bind to both cancer and malaria MDR pumps. The ATP-dependent MDR pump is inhibited because the ATPase involved in the reset mechanism for the pump is stimulated by tetrandrine as occurs similarly with verapamil in malaria and cancer cells. Therefore, the cells with multiple MDR pumps likely become energy depleted. It has been demonstrated that tetrandrine can inhibit the genetic mechanism that controls the induction or synthesis of the MDR exit pump. Therefore, it wouldn't be surprising tetrandrine can cure chloroquine-resistant falciparum malaria using similar mechanisms as found in MDR cancer cells.

Presently, it is understood that these MDR pumps cause the exit of fat soluble drugs rather than those substances that are highly hydrophilic or water soluble. Using this guideline, compounds of the structure ROR instead of ROH tend to be favored by these MDR exit pumps. Rather than trying to understand the structure-activity studies only in terms of greatly detailed stereochemistry alone, it is likely that the pump also recognizes the fat soluble chemical structures as substances that need to be pumped or excreted.

According to Fitch et al. [25], the major mechanism of action of chloroquine inside the malarial parasite is that chloroquine increases the amount of a regulator, which inactivates the catalyst for ferriprotoporphyrin 9 sequestration, which leads to accumulation of nonhemozoin ferriprotoporphyrin 9 , which binds with high affinity to chloroquine which is toxic to the parasite. This idea was originally stated that there is a polymerization of heme molecules which is inhibited by chloroquine could occur if a parasite heme polymerase were found. The idea that excessive free heme occurs due to drug action is quite likely correct. Sullivan et al. [26] created definitive evidence that chloroquine binds to heme forming a heme-quinoline complex which incorporates into the elongation site of the hemozoin polymer thus preventing further additions of heme and its elongation of the hemozoin polymer. Therefore, free heme builds up and kills the parasite.

If the parasite creates exit pumps to rid the chloroquine from itself, the chloroquine would partially cease to be an effective antimalarial. However, if an effective bisbenzyl isoquinoline (BBIQ) were added to chloroquine, the action of chloroquine is boosted more than 40 fold. How could this occur? The MDR exit pump requires ATP to effectively allow the pump to extrude the chloroquine from the parasite. But it also requires ATP via the action of ATPase to reset the pump. Our data [27] using purified MDR-ATPase demonstrates that tetrandrine can stimulate the action of ATPase, thus depleting the parasite of ATP. Other data from our group [27] indicates that the transcription mechanism which controls the production of the pump is inhibited by tetrandrine. This suggests that the remarkable synergism between chloroquine and tetrandrine occurs in part because the both production and action of the MDR exit pump is controlled by tetrandrine and similar compounds causing a buildup of heme and ultimately poisoning the parasite. The advantage of using a BBIQ/ chloroquine combination isn't just that it kills the parasite, an actual mechanism of resistance used by the malarial parasite is partially thwarted.
Other data discussed by Wellems and Plowe [28] indicates that a major mechanism involved in drug resistance to chloroquine is associated with a chloroquine transport mechanism which is inhibited by certain calcium channel blockers like verapamil. It is involved with a gene product pfcrt or (plasmodium falciparum chloroquine resistance transporter) which is localized on the food vacuole of the parasite. It is likely involved with the efflux of the drug away from it site of action to prevent chloroquine from working effectively to cause accumulation of heme to kill the parasite. Since calcium channel inhibitors are known to inhibit the Pfcrt and tetrandrine is a calcium channel inhibitor, it seems highly plausible that tetrandrine inhibits both major chloroquine resistance mechanisms. This may account for the 43 fold potentiation of chloroquine in the presence of tetrandrine in the plasmodium falciparum chloroquine resistant strain. The reversal becomes so striking that the combination of the two drugs in the chloroquine resistant strain is greater than the effects of chloroquine in the chloroquine sensitive strain!

Usually researchers constantly develop new antimalarials to combat malaria. This is a never ending struggle because the parasite develops simple or complex methods to become drug resistant. The MDR mechanism is an important mechanism to prevent antimalarial drugs from being effective. This method we have found should be effective for many hydrophobic or fat soluble antimalarial, parasitic or even bacterial drugs. We believe that this is a major finding that could apply to many anti-malarial, parasitic, bacterial or anticancer drugs developed in the future. In addition, many of the fundamental findings we have made with malarial parasites will also apply to many other parasites and bacteria and living cells that depend on the inducible MDR pump for protection against drugs developed by man. Furthermore, many of the BBIQs are relatively non-toxic drugs that have been used by the Chinese in their traditional medicine treatments for a variety of diseases for hundreds or thousands of years. We were the original developers of BBIQs to treat multiple drug resistant malaria and cancers as demonstrated in our original patents pending in 1989 and awarded in 1991 [29] and 1994 [30].

List of abbreviations of the bisbenzylisoquinolines and aporphine isoquinolines can be be found with Table1.

\section{Acknowledgements}

This investigation received financial support from the UNDP/World Bank/WHO Special Programme for Research and Training in Tropical Diseases (TDR). This grant award was M20/181/1 ID\#920304. One of us (ProfessorYe) received a fellowship from WHO. We gratefully acknowledge the financial support from the World Health Organization.

\section{This work was also supported by the following:}

1. Research Program of China Academy of Chinese Medical Science Exploratory study on new types of products about artemisinin-based antimalarial drugs. Fund Nos: ZZ0608 by Research Program of China Academy of Chinese Medical Science and

2. National Science and Technology Major Products for "Major new Drugs Innovation and Development" Study of methods for enhancing the efficacy and reducing the toxicity of compound compatibility Fund Nos: 2015ZX09501004-003-002 by National Science and Technology Major Products for "Major New Drugs Innovation and Development"

We thank Christopher Van Dyke for doing the organic structures for the various figures using the Chem Draw program. 
Citation: Ye Z, Van Dyke K (2015) Antimalarial Activity of Various Bisbenzylisoquinoline and Aporphine-Benzylisoquinoline Alkaloids and their Structure-Activity Relationships against Chloroquine - Sensitive and Resistant Plasmodium falciparum Malaria in vitro. Malaria Contr Elimination 5: 137. doi:10.4172/2470-6965.1000137

Page 7 of 7

\section{References}

1. Ye Z, Van Dyke K (1989) Selective antimalarial activity of tetrandrine against chloroquine resistant Plasmodium falciparum. Biochem Biophys Res Commun 159: 242-248.

2. Ye ZG, Van Dyke K, Castranova V (1989) The potentiating action of tetrandrine in combination with chloroquine or qinghaosu against chloroquine-sensitive and resistant falciparum malaria. Biochem Biophys Res Commun 165: 758-765.

3. Liu C, Xiao P (1983) Cepharanthine has Anticancer Effects Yaoxue Tongbao. Chem Abstr 18: 287-292.

4. Shiraishi N, Akiyama S, Nakagawa M, Kobayashi M, Kuwano M (1987) Effect of Bisbenzylisoquinolines (biscoclaurine) alkaloids on Multidrug Resistance in KB Cancer Cells. Cancer Research 47: 2413-2416.

5. Zhang Y (1987) Loci in Malaria vectors by genetic mapping. Parasitol 3: 190-192.

6. Kuroda H, Nakazawa S, Katagiri K, Shiratori C, Kozuka M, et al. (1976) Studies on the Chemical Constituents from Dehassia hainanesis. Chem Pharm Bull 24: 2413-2420.

7. Stoychkov JN (1980) Thalicarpine Increases the Anticancer Activity of Cyclophosphamide. Biomedicine 33: 42-47.

8. Herman EH, Chadwick DP (1973) Thalicarpine Affects the Cardiovascular System. Pharmacology 10: 178-197.

9. Xin WF, Xue Z (1983) Studies on the chemical constituents of Thalictrum omeiense. 18: 920-924.

10. Jia J, Zhu J, Yan H, Qian J, Xin W, et al. (1988) Methoxyadiantifoline produces a Chronotropic Effect on the Heart Chin. Pharmacol Bull 4: 237-240.

11. Hahn RA, Nelson JW, Tye A, Beal JL (2006) Pharmacological Activity of Methoxyadiantifoline. J Pharmaceutical Sciences 55: 466-469.

12. Reilly HB, Wang H, Steuter JA, Marx AM, Ferdig MT (2007) Quantitative dissection of clone-specific growth rates in cultured malaria parasites. Int J Parasitol 37: 1599-1607.

13. Desjardins RE, Canfield CJ, Haynes JC, Chubay J D (1979) Quantitative Assessment of Antimalarial Activity In Vitro by a Semi-automated Microdilution Technique Antimicrob. Agents Chemother 16: 710-718.

14. Carter GW, Van Dyke K (1971) A Superior Counting Solution for Water Soluble Tritiated Compounds. Clin Chem 17: 576-580.

15. Tallarida R, Murray R (1981) Manual of Pharmacologic Calculation 1: 19-20.

16. Cahn R, Ingold C, Prelog V (1966) Specification of Molecular Chirality Angewandte Chemie 5: 385-415.
17. Tolkachev ON, Vichkanova SA, Makarova LV (1978) Bisbenzylisoquiolines inhibit tubercle bacilli. Farmatsiya 27: 23-26.

18. Foote S, Thompson J, Cowman A, Kemp D (1989) Amplification of multiple drug resistance gene in some chloroquine resistance isolates of Plasmodium isolates. Cell 57: 921-930.

19. Ye Z, Van Dyke K, Spearman T, Safa AR (1989) 3H azidopine photoaffinity labeling of high molecular weight proteins in chloroquine resistant falciparum malaria. Biochem Biophys Res Commun 162: 809-813.

20. Partridge SJ, Russell PF, Anderson MM, Wright CW, Kirby GC, et al. (1988) In vitro antimalarial and antamoebic activity of protoberberine alkaloids. J Pharm Pharmacol 42: 47P.

21. Vichkanova SA, Makarova LV, Gordeikina NI (1973) Bisbenzylisoquinolines Inhibit Tuberculosis Bacilli. Chem Abstr 78: 106079.

22. King VF, Garcia ML, Himmel D, Reuben J, Lam YT, et al. (1988) Interaction of Tetrandrine with slowly inactivating calcium channels Characterization of calcium channel modulation by an alkaloid of Chinese medicinal origin. J Biol Chem 263: 2238-2244.

23. Ye ZG, Van Dyke K (1988) Reversal of chloroquine resistance in falciparum malaria independent of calcium channels. below Biochem Biophys Res Commun 155: 476-481.

24. Ye Z, Van Dyke K, Rossan RN (2013) Effective treatment with a tetrandrine/chloroquine combination for chloroquine-resistant falciparum malaria in Aotus monkeys. Malar J 12: 117.

25. Fitch, Coy D (1998) Involvement of Heme In the Antimalarial Action of Chloroquine, Transactions of the American Clinical and Climatological Association 109: 97-106.

26. Sullivan, David J, Gluzman, Ilya Y, Russell, et al. (1996) On the Molecular Mechanism of Chloroquine's Molecular Action. Proc Natl Acad Sci 93: 11865-11870.

27. Sosa, Arturo J, Chavez, Pedro, Van Dyke, et al. (2014) Inhibitory Effect of Tetrandrine and Paclitaxel or Doxorubicin On Multi-drug Resistant(MDR) Cancer Cells Associated With MDR -ATPase. International Journal of Phamacotherapy 4: 48-57.

28. Wellems TE, Plowe CV (2001) Chloroquine-resistant malaria. J Infect Dis 184: 770-776.

29. Van Dyke K (1989) Use of Tetrandrine and its Derivatives to Treat Malaria.

30. Van Dyke K (1994) Method for Potentiating Primary Drugs in Treating Multidrug Resistant Parasitic Disease Cells. 